\title{
Nazareth and Yezre'el Valley College (NYVC) study: Predictors of Stress, Anxiety and Depression among Pregnant Women During the First Wave of the COVID-19 Pandemic in Israel
}

\author{
Jimmy Jadaon ${ }^{1}$, Ola Ali-Saleh ${ }^{2}$, Ofra Halperin ${ }^{2}$, jawad karram ${ }^{1}$, Izhar Ben- Shlomo ${ }^{3}$, and \\ Liora Ore ${ }^{2}$ \\ ${ }^{1}$ EMMS Nazareth Medical Center \\ ${ }^{2}$ Max Stern Academic College Of Emek Yezreel \\ ${ }^{3}$ The Baruch Padeh Medical Center, Poriya. Affiliated to the Faculty of Medicine in the \\ Galilee. Bar-Ilan University Safed, Israel.
}

October 14, 2020

\begin{abstract}
We conducted a study that aimed to shed light on the effects of COVID-19 on the emotional state of pregnant and postpartum women, which are specifically vulnerable groups. A cross-sectional study based on a web questionnaire for the assessment of general emotional stressor during the quarantine period of COVID-19, from April 20th till May 7th. Overall, 356 women of whom 251 pregnant and 101 post-partum filled the questionnaire. Among 251 pregnant women, 199 (79.3\%) reported moderate-high stress, $145(57.8 \%)$ high anxiety. A hundred and three $(41.0 \%)$ reported a high level of depression (PH2>3). Among pregnant women, the following were the most prevalent stressors: fear that someone in their family will acquire the virus $(80.1 \%)$, concern for the health of the baby $(77.7 \%)$, fear that someone close will die from COVID-19 (72.9\%) and worry of being infected within the hospital $(71.7 \%)$. Univariate analysis of stress revealed that age $(\chi 2=10.93, \mathrm{p}<.004)$ and income level $(\chi 2=13.35, \mathrm{p}<.001)$ were associated with the level of stress. Stepwise logistic regression revealed that both age and income levels below the mean were predictors of stress. Among the 165 multiparous pregnant women, age (25-34 vs. 35-44) as a predictor of stress $(\mathrm{p}<.04)$. Our study clearly showed an enormous negative effect of the COVID-19 pandemic on the emotional condition of both pregnant and postpartum women. This provides an insight into the relation between economy and emotional state during the uncertainty period. This adds challenges to the healthcare system in providing means and solutions during disasters
\end{abstract}

\section{Hosted file}

BJOG - Manuscript.pdf available at https://authorea.com/users/367098/articles/486644nazareth-and-yezre-el-valley-college-nyvc-study-predictors-of-stress-anxiety-anddepression-among-pregnant-women-during-the-first-wave-of-the-covid-19-pandemic-in-israel 